\title{
Charge Regulation at Semiconductor-Electrolyte Interfaces
}

\author{
Mark E. Fleharty ${ }^{\mathrm{a}}$, Frank van Swol ${ }^{\mathrm{a}, \mathrm{b}}$, Dimiter N. Petsev ${ }^{\mathrm{a}}$ \\ ${ }^{a}$ Department of Chemical and Biological Engineering, University of New Mexico, Albuquerque, NM 87131 \\ ${ }^{b}$ Sandia National Laboratories, P.O. Box 5800, Albuquerque, NM 87185
}

\section{Abstract}

The interface between a semiconductor material and an electrolyte solution has interesting and complex electrostatic properties. Its behavior will depend on the density of mobile charge carriers that are present in both phases as well as on the surface chemistry at the interface through local charge regulation. The latter is driven by chemical equilibria involving the immobile surface groups and the potential determining ions in the electrolyte solution. All these lead to an electrostatic potential distribution that propagate such that the electrolyte and the semiconductor are dependent on each other. Hence, any variation in the charge density in one phase will lead to a response in the other. This has significant implications on the physical properties of single semiconductor-electrolyte interfaces and on the electrostatic interactions between semiconductor particles suspended in electrolyte solutions. The present paper expands on our previous publication [Phys. Rev. Lett 113 (2014) 158302] and offers new results on the electrostatics of single semiconductor interfaces as well as on the interaction of charged semiconductor colloids suspended in electrolyte solution.

\section{Introduction}

The interface between a semiconductor material and electrolyte solution presents a very interesting system from both a fundamental and applied perspective. Both the semiconductor and the electrolyte phases have mobile charges (electrons and/or holes in the former and ions in the latter) that can redistribute in space depending on the global potential distribution. Bringing these two phases in contact induces an electrostatic interaction between them via the boundary conditions at the interface. This leads to a redistribution of the local charges that form electric double layers in both the electrolyte and the semiconductor phases. The interface itself can have a complex chemical structure due to the presence of a native oxide layer that forms in contact with water, or due to deliberate surface functionalization [1-3]. This surface layer determines the groups that are exposed to the electrolyte solution and through the surface chemical equilibrium determines the charge at the interface. This is known as charge regulation [4-10] and defines the only physically justifiable boundary condition for solving such electrostatic problems. Hence, the electrostatic properties of the semiconductor-electrolyte interfaces are determined by the interplay between the charges in the two phases coupled to the specific interfacial chemistry. Any disturbance of the potential and charge density in one of the phases will induce a corresponding change in the other one. This has been experimentally demonstrated by Greenfield and Sivan [11] who developed a new type of force microscopy utilizing this effect. By measuring the conductivity variations in a semiconductor substrate these authors successfully measured the electrostatic interaction with an approaching charged object. Recently we performed a theoretical analysis of the interaction between two charged colloidal particles suspended in electrolyte solution [12]. It predicts that the electrostatic repulsion between the particles de- creases with increased doping level. Hence, doped semiconductor colloids suspended in electrolyte solution should be less stable than undoped ones if the surface chemical groups are identical for the two cases. The latter condition is important because if the doping procedure also changes the nature of the surface chemistry, the stability may be shifted in the opposite direction [13-15]. As the electrostatic repulsion drops with doping, attractive forces such as van der Waals can dominate and lead to particle coagulation $[16,17]$.

Colloidal systems that involve semiconductor-electrolyte interfaces or simply semiconductor particles suspended in electrolyte solutions are not only of significant fundamental interest but present opportunities for new applications $[2,18]$. The effect of charge density redistribution in the semiconductor in response to potential variations in the electrolyte near the interface has been exploited to perform force measurements [11, 19] and to develop sensing techniques that use nanoscale semiconductors [20-24].

This study is a continuation of our previous work [12], where we presented a semi-quantitative analysis of the stability of charged semiconductor colloids suspended in an electrolyte solution. Here we present a detailed analysis of the electrostatics of an isolated semiconductor-electrolyte interface, and thoroughly examine the effects of surface chemistry, salt concentration, and a wider range of doping concentrations abandoning several approximations made in the previous analysis. New results for highly-doped semiconductors are presented, and the role of the surface chemistry is investigated in more detail.

\section{Theory}

Consider the interface between a semiconductor material in contact with an electrolyte solution. Many semiconductors will form an oxide layer when in contact with an aqueous phase, the 
formation of silica on silicon being a typical example. The oxide layer provides chemical groups that are exposed to the electrolyte and may be involved in chemical reactions with some of the dissolved species. Often (and particularly when oxides are involved) the important species in solution are hydrogen ions which can bind to or dissociate from the oxide surface, which can be considered as ideally amphoteric. Unless specified otherwise, most of the model systems analyzed below are based on n-doped $\mathrm{Si}$ covered with a layer of $\mathrm{SiO}_{2}$.

\subsection{Potential Distribution at Semiconductor-Electrolyte Inter- faces}

While the surface groups may be involved in many chemical processes, most oxides can be adequately described by the following two chemical reactions [6]

$$
\begin{aligned}
\mathrm{AH}_{2}^{+} & \rightleftharpoons \mathrm{AH}+\mathrm{H}^{+}, \quad p \mathrm{~K}_{+}=-\log _{10} \mathrm{~K}_{+} \\
\mathrm{AH} & \rightleftharpoons \mathrm{A}^{-}+\mathrm{H}^{+}, \quad p \mathrm{~K}_{-}=-\log _{10} \mathrm{~K}_{-} .
\end{aligned}
$$

The $-A^{-}$groups are part of the solid material, typically the oxide. If the oxide layer is $\mathrm{SiO}_{2}$, then $-A^{-}=-\mathrm{SiO}^{-}$. The chemical equilibrium constants $K_{-}$and $K_{+}$determine the overall charge for a given value of the solution $p \mathrm{H}$. This surface charge gives rise to an electrostatic potential $\Psi$, which can be obtained by solving the Poisson equation

$$
\nabla^{2} \Psi=-\frac{\rho}{\varepsilon_{r} \varepsilon_{0}},
$$

where $\rho$ is the charge density, $\varepsilon_{r}$ is the relative dielectric permittivity of the local medium, and $\varepsilon_{0}$ is the permittivity of vacuum. The entire system, however, consists of a semiconductor region, oxide layer and electrolyte solution. Hence, Eq. (2) has to be solved in each of these domains. The charge density for each domain is given as

$$
\rho= \begin{cases}e \sum_{i} n_{i}^{0} z_{i} \exp \left(\frac{-z_{i} e \Psi}{k_{B} T}\right) & \text { electrolyte } \\ e N_{d}\left[1-\chi F_{1 / 2}\left(\frac{\mu-e \Psi}{k_{B} T}\right)\right] & \text { n-doped semiconductor } \\ 0 & \text { oxide }\end{cases}
$$

where $n_{i}$ is the number concentration of the $i^{\text {th }}$ ionic component, $z_{i}$ is the valence of the $i^{\text {th }}$ ionic component, $k_{B} T$ is the thermal energy, $e$ is the fundamental unit of charge,

$$
F_{1 / 2}(x)=\frac{2}{\sqrt{\pi}} \int_{0}^{\infty} d t \frac{t^{1 / 2}}{1+\exp (t-x)},
$$

is the Fermi integral of order $1 / 2$ and $\mu$ is the Fermi level, (i.e., the chemical potential of the charged species in the semiconductor) $[25,26]$. The parameter $\chi$ is given by,

$$
\chi=\frac{1}{4 N_{d}}\left(\frac{2 m^{*} k_{B} T}{\pi \hbar^{2}}\right)^{3 / 2},
$$

where $m^{*}$ is the effective mass of the electron and $\hbar$ is the reduced Planck constant. The charge density inside the oxide layer is assumed to be zero.
At high temperatures and moderate doping levels the Fermi integral can be approximated by an exponential function

$$
\chi F_{1 / 2}\left(\frac{\mu-e \Psi}{k_{B} T}\right) \rightarrow \exp \left(-\frac{e \Psi}{k_{B} T}\right) .
$$

This approximation breaks down below the Fermi temperature, which is defined by [27, 28]

$$
T_{F}=\frac{\hbar^{2}\left(3 \pi^{2} N_{d}\right)^{2 / 3}}{2 k_{B} m^{*}}
$$

Above the Fermi temperature, the Boltzman distribution is applicable, while deviation from the Boltzman distribution is noticeable below the $T_{F}$. Eq. (7) shows that the Fermi temperature increases with the doping level $N_{d}$, hence for heavily doped systems the approximation in Eq. (6) may break down. For example GaAs (with $m^{*}=0.067 m_{e}, m_{e}=9.11 \times 10^{-31} \mathrm{~kg}$ ) with doping level $N_{d}=10^{19} \mathrm{~cm}^{-3}$, exhibits a Fermi temperature of $T_{F} \approx 2900 \mathrm{~K}$ [27]. Similar calculations for Si with the same doping level $\left(m^{*}=0.28 m_{e}\right)$ gives $T_{F} \approx 700 \mathrm{~K}$. Hence, for higher doping levels the charge distribution in the semiconductor will be more accurately expressed by Eq. (3) rather than Eq. (6).

The surface charge density $\sigma_{s}$ at the oxide-electrolyte interface is simply defined as the sum of the positive charge density minus the negative charge density and is given as

$$
\sigma_{s}=e\left(\left[A H_{2}^{+}\right]-\left[A^{-}\right]\right),
$$

and the surface charge density can be related to the potential through

$$
\sigma_{s}=-\varepsilon_{r} \varepsilon_{0} \nabla \Psi \cdot \mathbf{n}
$$

at the surface where $\mathbf{n}$ is the vector normal to the interface pointing into the electrolyte.

Assuming the distribution of ions in the electrolyte obeys Boltzmann statistics, Eq. (8) can be expressed as a function of the surface potential $\Psi_{s}$ so that [6]

$$
\sigma_{s}\left(\Psi_{s}\right)=\frac{e \Gamma \delta \sinh \left[e\left(\Psi_{N}-\Psi_{s}\right) / k_{B} T\right]}{1+\delta \cosh \left[e\left(\Psi_{N}-\Psi_{s}\right) / k_{B} T\right]} .
$$

$\Gamma$ is the surface concentration of ionizable groups, $\delta=$ $2 \sqrt{K_{-} / K_{+}}$and the Nernst potential $\Psi_{N}$ is given as

$$
\Psi_{N}=\frac{k_{B} T}{e} \ln (10)(p \mathrm{I}-p \mathrm{H}) .
$$

Two important parameters that characterize the surface are the isoelectric point $p \mathrm{I}=\left(p \mathrm{~K}_{+}+p \mathrm{~K}_{-}\right) / 2$ and $\Delta p \mathrm{~K}=p \mathrm{~K}_{-}-p \mathrm{~K}_{+}$.

The boundary conditions required to solve Eq. (2), are to match potentials at the interfaces of the semiconductor (subscript "sc"), oxide (subscript "ox") and electrolyte (subscript "el") such that the charge regulating boundary condition is enforced at the oxide-electrolyte interface through,

$$
\Psi_{\mathrm{ox}}=\Psi_{\mathrm{el}}=\Psi_{s} ; \sigma_{s}=\mathbf{n} \cdot\left[\varepsilon_{\mathrm{ox}}(\nabla \Psi)_{\mathrm{ox}}-\varepsilon_{\mathrm{el}}(\nabla \Psi)_{\mathrm{el}}\right]
$$


while the boundary conditions applied at the semiconductoroxide interface are,

$$
\Psi_{\mathrm{sc}}=\Psi_{\mathrm{ox}} ; 0=\mathbf{n} \cdot\left[\varepsilon_{\mathrm{sc}}(\nabla \Psi)_{\mathrm{sc}}-\varepsilon_{\mathrm{ox}}(\nabla \Psi)_{\mathrm{ox}}\right]
$$

where $\sigma_{s}$ [see Eq. (8)] and $\Psi_{s}$ are the surface charge and potential governed by the chemical equilibria given in Eq. (1), and $\varepsilon_{\mathrm{sc}}, \varepsilon_{\mathrm{ox}}, \varepsilon_{\mathrm{el}}$ are the dielectric constants of the semiconductor, oxide and electrolyte respectively.

Solving Eqs. (2) and (3) subject to the boundary conditions given in Eqs. (10), (12) and (13) gives the distribution of the electrostatic potential $\Psi$ everywhere in the system. Certain geometries allow for obtaining an analytical solution of the nonlinear Poisson-Boltzmann equation [see Eq. (2) and the first line in Eq. (3)]. The solutions are well-known for the onedimensional equation for a single electric double layer [29], or two interacting electric double layers [16]. These simple geometries allow for obtaining formal solutions of the Poisson equation when the charge density is given by the Fermi integral [the second line in Eq. (3)] as we show in Appendix A. More complicated geometries (e.g., interacting spherical particles) allow for only numerical solution for the potential. In fact, because of the nonlinearity introduced by Eq. (10) practically every case will require a numerical step and it is often more practical to solve the entire boundary value problems using a numerical procedure.

\subsection{Interactions Between Two Charged Semiconductor Parti- cles in Electrolyte}

The osmotic pressure $\Pi_{e}$ due to the electric double layer between two semiconducting particles in an electrolyte is given as,

$$
\frac{\Pi_{e}}{n k_{B} T}=2\left[\cosh \left(\frac{z e \Psi}{k_{B} T}\right)-1\right]-\kappa^{-2}\left(\frac{z e \nabla \Psi}{k_{B} T}\right)^{2} .
$$

where $z$ is the valence of the background symmetric electrolyte, $n$ is the number concentration of the electrolyte and $\kappa^{-1}$ is the Debye Length

$$
\kappa^{-1}=\sqrt{\frac{\varepsilon_{r} \varepsilon_{0} k_{B} T}{2 z^{2} e^{2} n}} .
$$

By integrating at the midplane between two separated particles (see section 3.2 below) the energy of repulsion due to electrostatics is then determined through,

$$
U_{e}(h)=2 \pi \int_{h}^{\infty} d z \int_{0}^{\infty} d r \Pi_{e}(r, z) r
$$

and the van der Waals attractive interaction without taking retardation effects into account is then given by

$$
U_{\mathrm{vdw}}=-\frac{A_{H}}{6}\left\{\frac{2 a^{2}}{(h+2 a)^{2}}+\frac{2 a^{2}}{h^{2}+4 a h}+\ln \left[\frac{h(h+4 a)}{(h+2 a)^{2}}\right]\right\} .
$$

where $A_{H}$ is the Hamaker constant $[17,30,31]$. The total free energy is then given as $U=U_{e}+U_{\mathrm{vdw}}$.

\section{Results and Discussion}

\subsection{Single Interface}

The electrostatic potential distribution for an n-doped semiconductor-oxide-electrolyte system is shown in Fig. 1. It illustrates the effect of the doping level of the potential distribution in all phases. The oxide layer is charge-free and acts as a capacitor. The figure also demonstrates that the surface potential at the oxide-electrolyte interface varies moderately with doping. The effect of doping has a dramatic effect on the potential distribution inside the semiconductor because of screening, but a relatively small effect on the potential distribution in the electrolyte since the surface groups involved in charge regulation interact directly with the electrolyte. It is interesting to note however, that while doping seems to have only minor effect on the potential distribution in the electrolyte for a single interface, it plays a significant role if a second charged interface is present. As a second charged object approaches, the doping in the semiconductor enhances the lowering of the surface potential at the interface, thus reducing the overall repulsion (see Section 3.2 below).

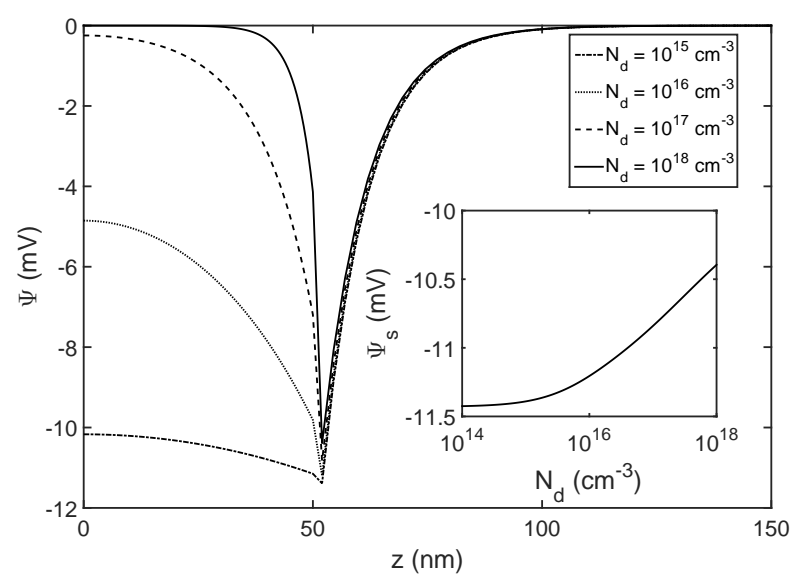

Fig. 1. Potential distributions plotted as a function of distance $z$. The semiconductor/oxide interface is at $z=50 \mathrm{~nm}$ while the oxide/electrolyte interface is at $z=52 \mathrm{~nm}$. The electrolyte has $p \mathrm{H}=3$. The various lines depict varying doping concentrations. The inset is a plot of surface potential $\Psi_{s}$ versus doping $N_{d}$.

In contrast to the doping effect, varying the electrolyte concentration significantly changes the electrostatic potential everywhere (see Fig. 2). The drop of the potential with the electrolyte concentration is very pronounced. The reason for this strong effect is due to the fact that the charge regulating surface groups are exposed to the electrolyte solution and chemically interact with the potential determining ions there [see Eq. (1)]. The surface charge $\sigma_{s}$ is strongly affected by changes in the electrolyte such as potential screening due to the variation of the salt concentration, which leads to a change in the potential distribution also in the semiconductor phase (across the capacitive oxide layer).

Fig. 3 illustrates the effect of the surface chemistry on the electrostatic potential distributions. The surface chemistry ef- 


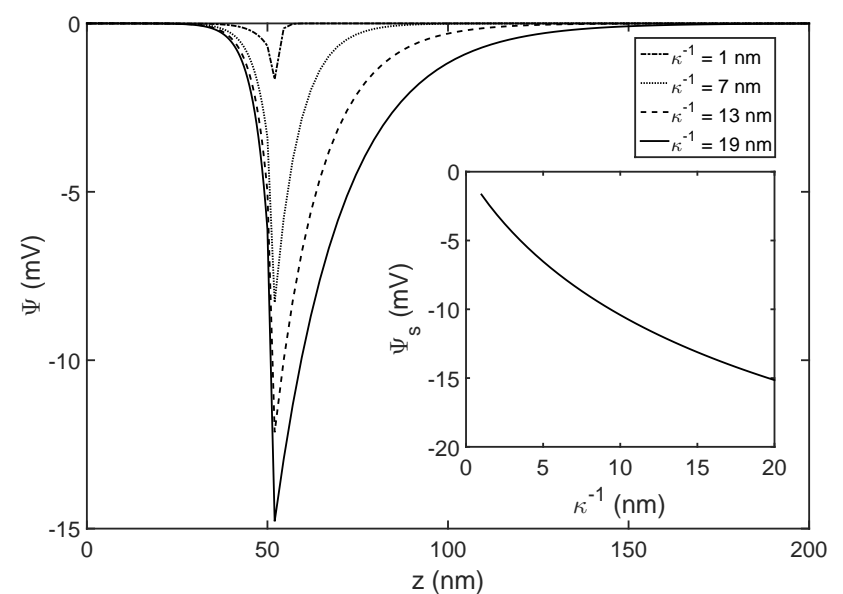

Fig. 2. Potential distributions plotted as a function of distance $z$. The parameters are the same as those given in Figure 1 but at constant doping of $N_{d}=10^{18} \mathrm{~cm}^{-3}$. The inset is a plot of the surface potential $\Psi_{s}$ versus Debye length $\kappa^{-1}$.

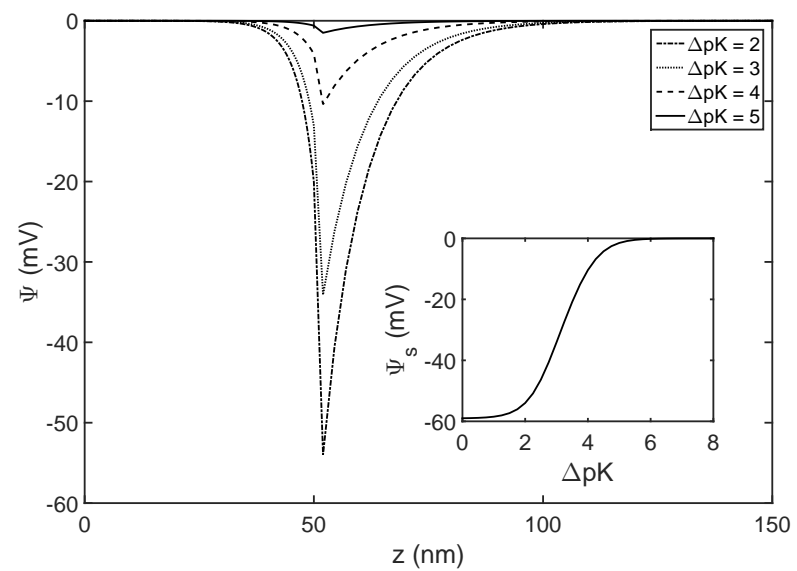

Fig. 3. Potential distributions plotted as a function of distance $z$ for different values of $\Delta p \mathrm{~K}$. The parameters are the same as those given in Figure 1 but at constant doping of $N_{d}=10^{18} \mathrm{~cm}^{-3}$. The inset shows surface potential versus $\Delta p \mathrm{~K}$.

fect is complex and depends on the chemical structure of the interface as well as on the composition of the solution. The surface groups have an affinity to particular ionic species (the potential determining ions, in this case $\mathrm{H}^{+}$) and the main characteristic of this interaction is quantified by the equilibrium constants or the $\Delta p \mathrm{~K}$ values [see Eq. (1)]. The difference between $\Delta p \mathrm{~K}$ in conjunction with the $p \mathrm{H}$ determines how charged the surface would be as a result of the chemical reactions in Eq. (1). That is why the electrostatic potential distributions in both the semiconductor and electrolyte phases are strong functions of $\Delta p \mathrm{~K}$. Varying the surface chemistry and hence, the value of $\Delta p \mathrm{~K}$ is possible only by deliberate surface modification, and might be an experimentally non-trivial task for certain materials. Still, it is interesting to know how the global electrostatics are impacted by the specific chemical groups exposed at the interface with the electrolyte solution. The results shown in Fig. 3 suggest that as $\Delta p \mathrm{~K}$ decreases, the magnitude of electrostatic potential in all phases increases. It is important to note that the $p \mathrm{H}$ of the solution is one unit higher than the isoelectric point, which is the same for all cases, i.e., $p \mathrm{I}=2$ which is approximately that of silica [32]. Note that for $\Delta p \mathrm{~K}=2, p \mathrm{H}=p \mathrm{~K}_{-}$. For $\Delta p \mathrm{~K}=0$ the surface potential becomes $\Psi_{N}=\Psi_{s} \approx 60 \mathrm{mV}$. In the other extreme when $\Delta p \mathrm{~K}$ is large, the magnitude of the surface charge tends to zero. The reason is that for large $\Delta p \mathrm{~K}$, the values of $p \mathrm{~K}_{-}$and $p \mathrm{~K}_{+}$are so far away from the solution $p \mathrm{H}=3$ that any of the reactions in (1) tend towards the neutral species.

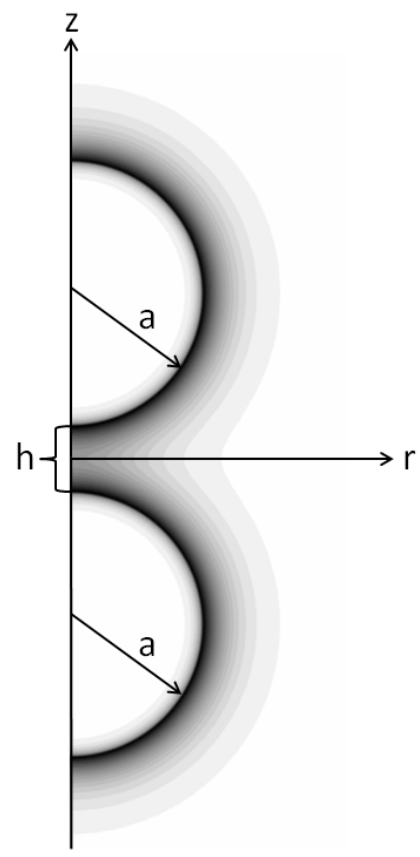

Fig. 4. Visualization of system consisting of two particles. Axis symmetry along $z$ with the other spatial coordinate $r$ results in the two particles being spherical in geometry. The separation of the particles is given as $h$, and the radius of the particles is $a$. The shaded portions represent the magnitude of the potential $\Psi$ for a particular set of values, darker shades indicate larger values. It can be seen that the smallest values of $\Psi$ are far from the particle surface in the electrolyte, as well as deep in the interior of the semiconductor particles. At this scale the oxide layer is too thin to be easily seen.

\subsection{Interaction between Semiconductor Colloidal Particles in Electrolyte Solution}

Knowing the electrostatic potential distribution we can obtain the interaction energy between two semiconductor particles as described above in section 2.2 (See Figs 4 and 5). We have obtained an energy plot in our previous work [12] using the so-called Derjaguin approximation [17, 30, 31], which is valid for particles with radius greater than the separation distance between them. Here we relax this limitation, by numerically integrating the disjoining pressure over the midplane separating the particles at different antiparticle separation [see Eq. (14) and Fig. 4] to obtain the force vs distance relationship. Next 
we integrate the force over the distance to obtain the electrostatic energy of interaction. The van der Waals contribution given by Eq. (17) is added to it to obtain the total energy of interaction. The parameters for the computation are Hamaker constant $A_{H}=5.4 \times 10^{-20} \mathrm{~J}$, particle radius $a=100 \mathrm{~nm}$, density of the surface charged groups $\Gamma=8 \times 10^{18} \mathrm{~m}^{-2}$, electrolyte concentration $0.925 \mathrm{mM}, p \mathrm{~K}_{+}=-2, p \mathrm{~K}_{-}=6$, particle doping $10^{18} \mathrm{~cm}^{-3}$ and dielectric permittivities $\varepsilon_{\mathrm{Ox}}=3.9, \varepsilon_{\mathrm{el}}=78.5$, and $\varepsilon_{\mathrm{sc}}=11.7$. The thickness of the oxide layer is $2 \mathrm{~nm}$. The numerically obtained energy curve is very similar to the one derived using Derjaguin approximation [12]. However, there are some important differences. The Derjaguin approximation tends to overestimate the difference between the doped and undoped cases, as well as the magnitude of the secondary minimum at larger separations. Despite the reduction, there is still a significant difference between the energy barriers for the doped and undoped cases, which will have a significant effect on the stability [12]. The inset to Fig. 5 shows the difference between interaction energies for two n-doped, two p-doped and one ndoped particle interaction with a p-doped one. The differences are due to the fact that the different mobile charges in the semiconductors will interact slightly differently with the always negatively charged interface. However, the main effects on the total interaction energy is primarily due to the charge redistribution and much less to its polarity. Fig. 6 demonstrates the effect of

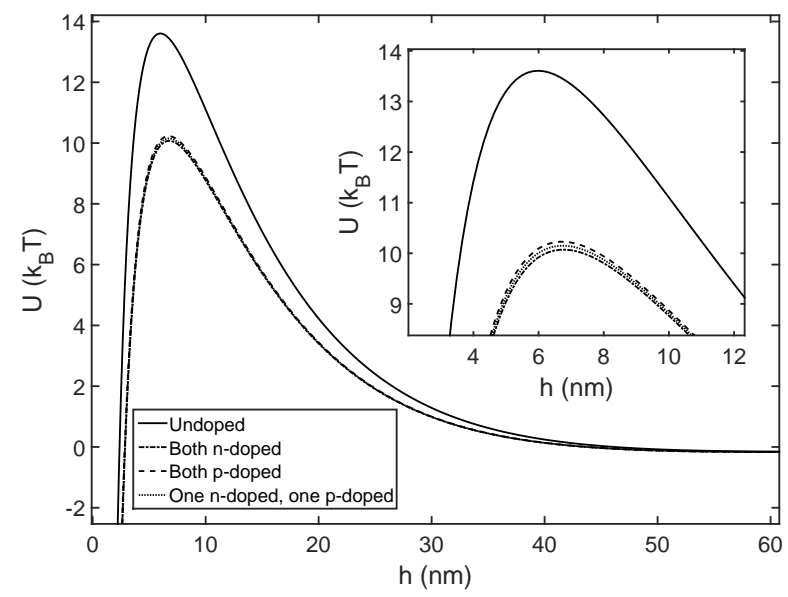

Fig. 5. Interaction energy versus separation $h$. The solid line is for two undoped particles, a noticeable reduction in free energy can be seen for the various combinations of doped particle interactions. The inset provides a closer look at the peak of the doped particle interaction energies.

the surface chemistry by plotting the energy-distance curves at different values for the parameter $\Delta p \mathrm{~K}$. Larger values of $\Delta p \mathrm{~K}$ reduce the energy of interaction and vice versa. These results are in agreement with those shown in Fig. 3. The isoelectric point of the surface is again $p \mathrm{I}=2$ and the $p \mathrm{H}=3$. As $\Delta p \mathrm{~K}$ is increased the surface charge on both particles goes down, thus reducing the repulsion. Hence, the surface chemistry has a strong effect in the interaction energy between semiconductor colloids, very much like it has on interaction energy between dielectric particles in suspension. Still, there is a difference be- tween doped and undoped particles. A greater $\Delta p \mathrm{~K}$ value will generally destabilize doped particles mare than undoped.

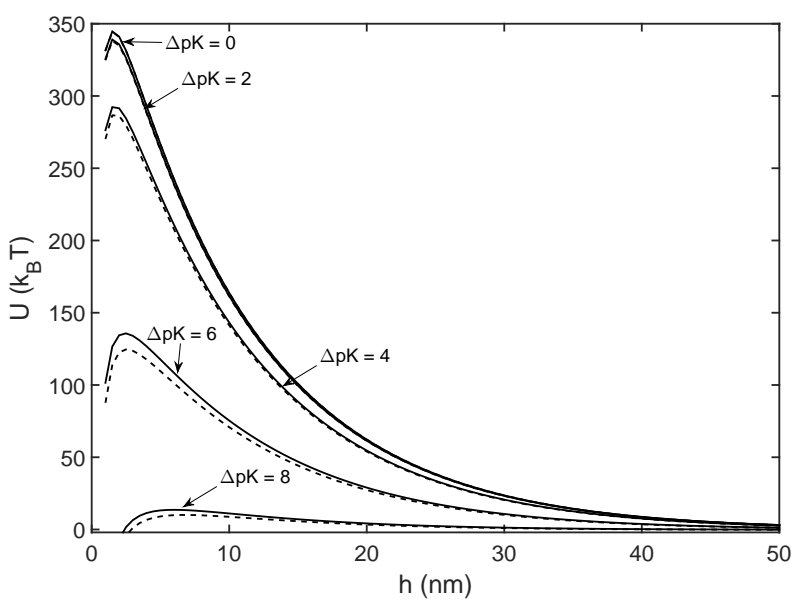

Fig. 6. Interaction energy versus separation $h$. The solid lines are for undoped spherical particles and the dashed lines are for their doped counterparts where $N_{d}=10^{18} / \mathrm{cm}^{3}$.

For highly doped systems where $T_{F}>T$ the approximation to Fermi statistics using an exponential as described in Eq. (6) breaks down. Fig. 7 illustrates how the potential distribution inside the semiconducting region is affected by the exponential approximation versus using the Fermi integral expression for the charge density distribution [see the second line in Eq. (3)]. Details explaining the solution procedure are given in the Appendix. At moderate doping concentrations of $N_{d}=10^{18}$ the potential distributions are very close, and as doping is decreased the exponential approximation is a very good one. However, at larger doping concentrations it can be seen that it is necessary to use the Fermi integral in the evaluation of the potential distribution. It interesting to point that the exponential (Boltzmann) approximation for doping level equal to $N_{d}=10^{19}$ is almost identical to the more accurate result based on the Fermi model for $N_{d}=10^{20}$. Hence, the approximate Boltzmann expression is completely inadequate at high levels of doping. The use of the Fermi integral at high doping also reveals that the reduction in the magnitude of the surface potential $\Psi_{s}$ is less than predicted by the exponential approximation.

\section{Conclusion}

Doped semiconductor-electrolyte interfaces exhibit properties that differ from their dielectric counterparts. These differences are due to the communication between charge carriers in the semiconductor, and electrolyte phases. In many cases this communication takes place across an oxide layer that covers the particle. The role of the layer can be important as it determines the surface chemistry and interfacial charge regulation. The communication between these phases is maintained by balancing the charge and potential at all interfaces.

The main feature that distinguishes a semiconductor colloid from an regular dielectric particle is the presence of mobile 


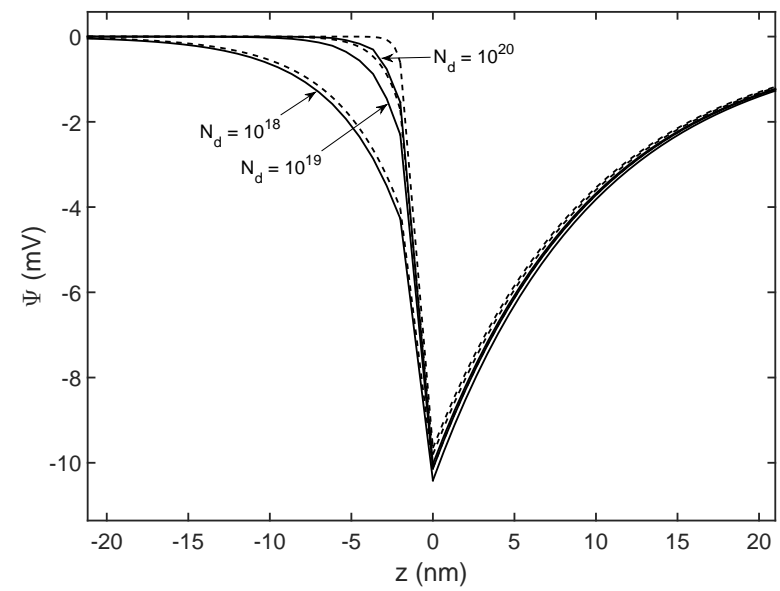

Fig. 7. Potential distributions one dimensional semiconductor-oxide-electrolyte system demonstrating the difference between the approximation described in Eq. (6). The dashed lines were calculated using Boltzmann statistics while the full lines were calculated by numerically evaluating the Fermi integral in Eq. (3).

charges in its interior. In general, the effect of charge mobility in the semiconductor region is to reduce the electrostatic potential at the interface. This in turn results in a reduction in the overall energy of interaction between doped semiconductor particles in electrolyte. We show that the reduction of the surface potential is primarily due to mobile charge density in the semiconductor, which responds to perturbations in the global electrostatic potential. The particular type of these charges ( $p$ doped or n-doped) has relatively little effect. For this reason, when two semiconductor particles are present in solution, the doping will reduce their stability if both are n-doped, p-doped, or even if one particle is n-doped, and the other is p-doped.

The surface chemistry and properties of the electrolyte including salt concentration and $p \mathrm{H}$ are extremely important. They have significant effect on the surface charge magnitude and type. In the case of doped semiconductor colloids, this effect couples to the effect of redistribution of the internal charge density.

The effect described in this study have a significant impact on the stability behavior of suspensions. It can also be exploited for a variety of applications related to surface force measurements, or chemical and biochemical sensing.

Acknowledgements : We thank Dr. James E. Miller for reading the manuscript and making many useful comments and suggestions. This research supported by NSF (CBET 0844645) and the United States Department of Energy, Office of Basic Energy Sciences, Division of Materials Sciences and Engineering. Sandia National Laboratories is a multi-program laboratory managed and operated by Sandia Corporation, a wholly owned subsidiary of Lockheed Martin Corporation, for the U.S. Department of Energys National Nuclear Security Administration under contract DE-AC04-94AL85000.

Mark Fleharty was also supported by the UNM Graduate Excellence Fellowship, GAANN (P200A090028), and the Char- lotte and William Kraft Graduate Fellowship. We are also thankful to the UNM Center for Advanced Research Computing for computational resources used in this research.

\section{Appendix A. Formal Solutions for the Poisson Equation with Charge Density Given by the Fermi In- tegral}

Our derivation below is for the particular case of $n$-doped semiconductor where the mobile charges are electrons, which is in contact with electrolyte solution across a thin oxide layer. The result for p-doped would be similar. The equation that we need to solve is

$$
\frac{d^{2} \tilde{\Psi}}{d \tilde{x}^{2}}=-\left[1-\chi F_{1 / 2}(\tilde{\mu}-\tilde{\Psi})\right]
$$

where we have adopted the notation $\tilde{\Psi}=e \Psi / \varepsilon_{r} \varepsilon_{0} k_{B} T, \tilde{\mu}=$ $\mu / k_{B} T, \tilde{x}^{2}=x^{2} e^{2} N_{d} / \varepsilon_{r} \varepsilon_{0} k_{B} T$. The Eq. (A.1) is multiplied by $2\left(d^{2} \tilde{\Psi} / d \tilde{x}^{2}\right)$ and integrated. For a single electric double layer the potential and its derivative vanish far away from the interface and the result is

$$
\frac{d \tilde{\Psi}}{d \tilde{x}}=\left\{2 \chi\left[F_{3 / 2}(\tilde{\mu}-\tilde{\Psi})-F_{3 / 2}(\tilde{\mu})\right]-2 \tilde{\Psi}\right\}^{1 / 2}
$$

where

$$
F_{3 / 2}(x)=\frac{1}{\Gamma(5 / 2)} \int_{0}^{\infty} d t \frac{t^{3 / 2}}{1+\exp (t-x)},
$$

is a Fermi integral of order $3 / 2$. A second formal integration leads to

$$
\int_{\tilde{\Psi}_{0}}^{\tilde{\Psi}} \frac{d \Psi}{\left\{2 \chi\left[F_{3 / 2}(\tilde{\mu}-\tilde{\Psi})-F_{3 / 2}(\tilde{\mu})\right]-2 \tilde{\Psi}\right\}^{1 / 2}}=\tilde{x} .
$$

The surface potential $\tilde{\Psi}_{0}$ is defined at the semiconductoroxide interface and has to be determined from the boundary conditions given in Eq. (12) and Eq. (13).

\section{Appendix B. Determining the Fermi Level for a Single Semiconductor-Oxide-Electrolyte Interface}

The value of the Fermi level (i.e., the chemical potential of the electrons) is necessary to know when the Boltzmann approximation (6) fails and the Fermi-Dirac distribution has to be used in the semiconductor (3). The determination of the Fermi level depends on the system. If transfer of charge (in this case an electron) is allowed, the Fermi level in the semiconductor would be fixed through an appropriate boundary condition at the interface with the adjacent phase [27]. If electron transfer between the phases is not possible, the Fermi level can be obtained from the condition for charge conservation [26]. A particularly simple case is when the semiconductor phase is large 
enough so that the total charge due to donors and free electrons far away from the charged interface becomes zero. At this point the electrostatic potential will also vanish and the Fermi level can be obtained through the charge neutrality condition

$$
1-\chi F_{1 / 2}\left(\frac{\mu}{k_{B} T}\right)=0
$$

This is the equation we use to find the Fermi level for calculating the properties of a single semiconductor-oxideelectrolyte interface above. The value of $\mu$ depends only on the parameter $\chi$ [see Eq. (5)]. Fig. B.8 shows the dependence of the Fermi level on the parameter $\chi$ obtained by solving Eq. (B.1).

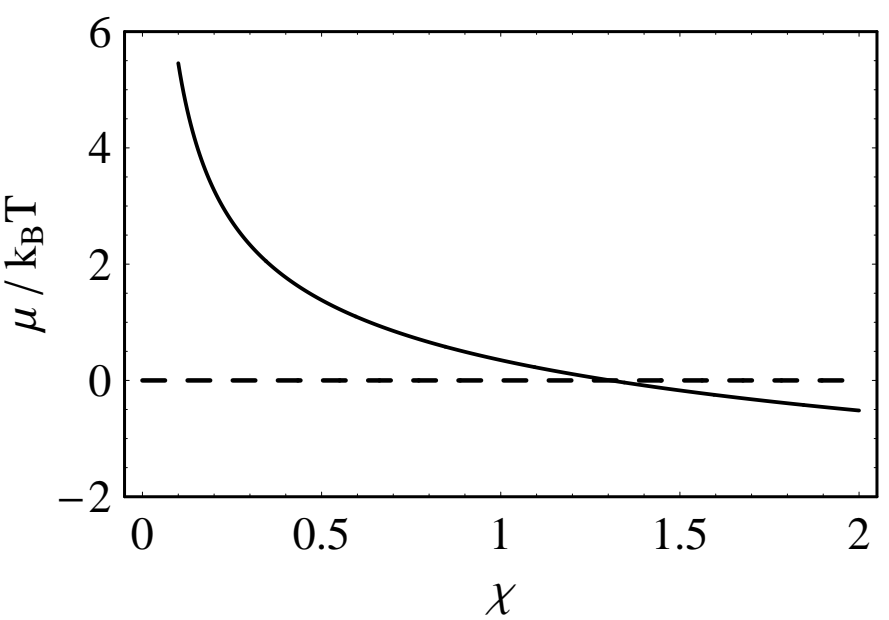

Fig. B.8. Fermi level $\mu$ vs the parameter $\chi$.

[1] M. V. Kovalenko and M. Scheele and D. V. Talapin, Science 324 (2009) $1417-1420$

[2] D. V. Talapin and J.-S. Lee and M. V. Kovalenko and E. V. Shevchenko, Chem. Rev. 110 (2010) 389-458.

[3] A. Nag and Maksym V. Kovalenko and Jong-Soo Lee and Wenyong Liu and Boris Sokoyny and Dimitri V. Talapin, J. Am. Chem. Soc. 133 (2011) 10612-10620.

[4] B. W. Ninham and V. A. Parsegian, J. Theor. Biol. 31 (1971) 405-428.

[5] S. L. Carnie and D. Y. C. Chan, J. Colloid Interface Sci. 161 (1993) 260264.

[6] D. Y. C. Chan and T. W. Healy and L. R. White, J. Chem. Soc., Faraday Trans. I 72 (1976) 2844-2865.

[7] D. Y. C. Chan, T. W. Healy, T. Supasiti and S. Usui, J. Colloid Interface Sci. 296 (2006) 150-158.

[8] S. H. Behrens and M. Borkovec, J. Chem. Phys. 111 (1999) 382-385.

[9] S. H. Behrens and M. Borkovec, J. Phys. Chem. 103 (1999) 2918-2928.

[10] S. H. Behrens and M. Borkovec, Phys. Rev. E 60 (1999) 7040-7048.

[11] E. Greenfield and U. Sivan, Phys. Rev. Lett. 102 (2009) 106101.

[12] M. E. Fleharty and F. van Swol and D. N. Petsev, Phys. Rev. Lett 113 (2014) 158302
[13] H. Sugimoto and M. Fujii and K. Imakita and S. Hayashi and K. Akamatsu, J. Phys. Chem. C 116 (2012) 17969-17974.

[14] H. Sugimoto and M. Fujii and K. Imakita and S. Hayashi and K. Akamatsu, J. Phys. Chem. C 117 (2013) 6807-6813.

[15] H. Sugimoto and M. Fujii and K. Imakita and S. Hayashi and K. Akamatsu, J. Phys. Chem. C 117 (2013) 11850-11857.

[16] E. J. W. Verwey and J. Th. G. Overbeek, Theory of the Stability of Lyophobic Colloids, Elsevier Publishing Company, Inc., 1948.

[17] B. V. Derjaguin and N. V. Churaev and V. M. Muller, Surface Forces, Plenum, 1987.

[18] F. Meseguer and R. Fenollosa and I. Rodriguez and E. Xifré-Perez and F. Ramiro-Manzano and M. Garín and M. Tymczenko, J. Appl. Phys. 109 (2011) 102424.

[19] S. Lekkala and J. A. Marohn and R. F. Loring, J. Chem. Phys. 139 (2013) 184702.

[20] Y. Cui and Q. Wei and H. Park and C. M. Lieber, Science 293 (2001) 1289-1292.

[21] J.-L. Hahm and C. M. Lieber, Nano Lett. 4 (2004) 51-54.

[22] Fernando Patolsky and Brian P. Timko and Guihua Yu and Ying Fang and Andrew B. Greytak and Gengfeng Zheng and Charles M. Lieber, Science 313 (2006) 1100-1104.

[23] Fernando Patolsky and Gengfeng Zheng and Oliver Hayden and Melike Lakadamyali and Xiaowei Zhuang and Charles M. Lieber, Proc. Natl Acad. Sci. (USA) 101 (2004) 14017-14022.

[24] Y. Zhang and T. C. Gamble and A. Neumann and G. P. Lopez and S. R. Brueck and D. N. Petsev, Lab on a Chip 8 (2008) 1671-1675.

[25] R. P. Feynman and N. Metropolis and E. Teller, Phys. Rev. 75 (1949) 1561.

[26] R. Kubo, Statistical mechanics, an advanced course with problems and solutions, North-Holland Personal Library, 2004.

[27] J. H. Luscombe and A. M. Bouchard and M. Luban, Phys. Rev. B 46 (1992) 10262-10268.

[28] W.-D. Kraeft and D. Kremp and W. Ebeling and G. Ropke, Quantum statistics of charged particle systems, Akademie-Verlag, Berlin, 1986.

[29] G. Gouy, J. Physique 9 (1910) 457-468.

[30] W. B. Russel and D. A. Saville and W. R. Schowalter, Colloidal Dispersions, Cambridge Univseristy Press, 1989.

[31] J. N. Israelachvili, Intermolecular and surface forces. 2nd edn, Academic Press, 2011.

[32] R. E. G. van Hal and J. C. T. Eijkel and P. Bergveld, Adv. Coll. Interf. Sci. 69 (1996) 31-62. 